\title{
Propuesta de manejo mejorado de pasturas en la economía de ganaderos de la sierra norte de Perú
}

\section{Proposal for improved pasture management in the livestock economy of the northern highlands of Peru}

\author{
Luis Vallejos Fernández ${ }^{1 *}$ (D) Jorge Álvarez Giambruno² ${ }^{2}$
}

\section{RESUMEN}

La actividad lechera constituye el sustento económico de un importante número de familias en la sierra norte del Perú. Sus resultados económicos se han visto afectados por el bajo rendimiento promedio de leche por vaca en lactancia. Este estudio se realizó con el objetivo de plantear una propuesta que permita incrementar los resultados productivos y económicos de los productores de la sierra norte del Perú, a partir de una mejora en el manejo de pasturas tradicional. El estudio contrasta el manejo tradicional con una propuesta que hemos denominado manejo mejorado. Esta comparación se realiza sobre la base de una hectárea de terreno utilizada para el pastoreo de ganado en producción. La escasa fertilización de los suelos, frecuencias de pastoreo de 70 días a más, y nula conservación de forrajes, serán reemplazados por fertilización de acuerdo a las necesidades identificadas por análisis químico del suelo, frecuencias de pastoreo de 40 días, renovación de pasturas a través de la resiembra y conservación del forraje excedente durante la época de lluvia para ser utilizado como heno en la época de estiaje. Las áreas de suelo resembradas y fertilizadas incrementan en $100 \%$ la producción de pastos, por otro lado, utilizando frecuencias de pastoreo menores a 70 días se obtienen una mayor concentración de nutrientes y se reduce el porcentaje de malezas. Se concluye que la propuesta genera un resultado económico que permite tanto, financiar las inversiones necesarias, como aumenta los niveles de ingreso de los productores.

Palabras clave: producción lechera, mejoramiento forrajero, evaluación tecnológica.

\begin{abstract}
Dairy farming constitutes the economic main activity of a significant number of families in the northern highlands of Peru. Their economic results have been affected by the low average yield of milk per milking cow. This study was carried out with the objective of establishing a proposal that allows increasing the productive and economic results of farmers in the northern highlands of Peru, based on an improvement in traditional pasture management. The study contrasts traditional pasture management with a proposal that we have called improved pasture management. This comparison is made based on one hectare of land used for grazing milking livestock. The scarce fertilization of the soils, grazing frequencies of 70 days or more, and no conservation of forages characterize traditional pasture management. This will be replaced by fertilization according to the needs identified by chemical analysis of the soil, grazing frequencies of 40 days, renewal of pastures through of the replanting and conservation of the surplus forage during the rainy season to be used as hay in the dry season. Replanted and fertilized areas of soil increase pasture production by $100 \%$, on the other hand, using grazing frequencies of less than 70 days, the concentration of nutrients is greater and the percentage of weeds is reduced. It is concluded that the proposed improvement generates an economic result that allows financing the necessary investments and increases the income levels of the farmers.
\end{abstract}

Keywords: milk production, forage improvement, technological evaluation.

\footnotetext{
${ }^{1}$ Universidad Nacional de Cajamarca, Facultad de Ingeniería en Ciencias Pecuarias, Cajamarca, Perú ${ }^{2}$ Universidad de la República, Facultad de Agronomía, Montevideo, Uruguay.

"Autor de Correspondencia. E-mail: lvallejos@unc.edu.pe
} 


\section{INTRODUCCIÓN}

Las pasturas, base de la alimentación del ganado bovino lechero de Cajamarca, están representadas por la asociación raigrás (Lolium multiflorum) "ecotipo cajamarquino" y el trébol blanco (Trifolium repens) variedad Ladino. Introducidas estas especies, desde mediados del siglo pasado, han constituido hasta hoy, la fuente principal de nutrientes para el mantenimiento, crecimiento y producción del ganado lechero existente en la sierra norte del Perú. Estas pasturas se naturalizaron y establecieron gracias a las favorables condiciones de clima y temperatura del medio, así como también a la frecuencia de pastoreo en estado reproductivo (mayor a 70 días) que permite a las semillas ser resembradas naturalmente, a través del pisoteo o consumo de los animales. Los productores de leche no consideran, en su mayoría, importante la fertilización, basados probablemente en que las excretas de sus animales devuelven los nutrientes al suelo. Sin embargo, pueden ocurrir impactos negativos en la fertilidad del suelo (Silveira y Kohmann, 2020), debido a que un suelo deficiente en nutrientes, genera plantas carentes de nutrientes, y por tanto estiércol deficiente en dichos nutrientes (Marino y Agnusdei, 2004). En pasturas asociadas al raigrás trébol, uno de los minerales más influyentes en la producción (Álvarez, 2006) y composición botánica (García, 2006) es el fósforo. En la sierra norte del Perú, a mayor altitud sobre el nivel del mar, el fósforo (P) presenta niveles más bajos de concentración en el suelo. Entre 2300 a 2800 m.s.n.m. es de 5,5 ppm; de 2801 a 3300 m.s.n.m., de 1,7 ppm; y entre 3301 a 3800 m.s.n.m., 1,9 ppm (Vallejos, 2017). En estas condiciones, el uso de fósforo en nivel de $80 \mathrm{~kg} / \mathrm{ha}$ incrementa de manera significativa el rendimiento de forraje (Vallejos, 2009).

La frecuencia de pastoreo, expresada como el tiempo que transcurre desde que los animales salen de un potrero y regresan al mismo, varía de 60 a 80 días en la sierra norte del Perú. En este tiempo, las características nutricionales, morfológicas y entorno (composición botánica) de la planta, se modifican afectando el consumo, rendimiento y tasa de crecimiento del forraje (Vallejos, 2009). La frecuencia de pastoreo de 70 días a más, influye en la composición botánica de las pastura. En este sentido, el trébol blanco se ve afectado por ser una especie leguminosa rastrera, que ve su crecimiento, reducido debido a la falta de luz solar que la hace fotosintéticamente menos activa ( $\mathrm{Yu}$ Ying y Hou Fu, 2005), disminuyendo su presencia en las pasturas asociadas reduciéndose a niveles de 1,0\% (Vallejos et al., 2019). De la misma manera, la presencia de especies no deseables (Pennisetum clandestinum, Taraxacum officinale, Rumex cripus) como parte de la composición florística representa porcentajes de $36 \%$ (Vallejos et al., 2019) a $45 \%$ en la mayoría de predios de Cajamarca. Si a esto se añade el bajo valor nutricional, con un 9,95\% de proteína cruda (PC) (Carrasco, 2019) y aprovechamiento ineficiente del potencial de producción de pasto durante la época de lluvia, para ser conservados como heno y utilizado durante la época de estiaje, justifican claramente la baja producción de leche del ganado (5 kg leche/vaca) (Vallejos, 2009) y los pobres ingresos de los pequeños y medianos productores. Es así que, suplementando a los animales durante la época de estiaje con forrajes conservados, pasturas resembradas y fertilizadas, con frecuencia de pastoreo de 40 días, la producción de leche diaria se incrementa entre el $60 \%$ al $80 \%$ (Vallejos, 2011).

A partir de los datos aportados por la investigación nacional, y de observar las prácticas productivas desarrolladas por algunos productores innovadores, se ha elaborado una propuesta de manejo mejorado que pretende salir del actual estancamiento que resulta en bajos rendimientos de producción forrajera, mala nutrición de las vacas lecheras, baja producción de leche, sobrepastoreo y malos resultados económicos que comprometen la calidad de vida de las familias ganaderas (Perinango, 2019) y la capacidad de realizar mejoras en el sistema. El objetivo del presente estudio es proponer una mejora en el manejo de pasturas que permita incrementar su producción, calidad, disponibilidad y rendimiento lácteo del ganado, beneficiando 
económicamente a los ganaderos lecheros. Esta propuesta será evaluada comparándola con el manejo tradicional que realizan los productores lecheros de la sierra norte del Perú.

\section{MATERIALES Y MÉTODOS}

Las condiciones favorables del clima durante el año
(Tabla 1), dada la ubicación geográfica de Cajamarca (Latitud 06 $48^{\prime} 00^{\prime \prime}$ S y Longitud 78 $48^{\prime} 00^{\prime \prime} \mathrm{W}$ ) ubicada en la sierra norte del Perú, representan una importante opción de éxito de la propuesta que se plantea en beneficio de las familias rurales.

Tabla 1. Temperatura y precipitación pluvial promedio en tres altitudes de Cajamarca. (PA I = 2300 a 2800 m.s.n.m.; PA II = 2801 a 3300 m.s.n.m., PA III = 3301 a 3800 m.s.n.m.)

\begin{tabular}{|c|c|c|c|c|c|c|}
\hline \multirow[b]{2}{*}{ Mes } & \multicolumn{2}{|c|}{ PAI } & \multicolumn{2}{|c|}{ PAII } & \multicolumn{2}{|c|}{ PAIII } \\
\hline & $\mathrm{T}^{\circ}\left({ }^{\circ} \mathrm{C}\right)$ & $\begin{array}{l}\text { Precipitación } \\
(\mathrm{mm})\end{array}$ & $\mathrm{T}^{\circ}\left({ }^{\circ} \mathrm{C}\right)$ & $\begin{array}{l}\text { Precipitación } \\
(\mathrm{mm})\end{array}$ & $\mathrm{T}^{\circ}\left({ }^{\circ} \mathrm{C}\right)$ & $\begin{array}{l}\text { Precipitación } \\
(\mathrm{mm})\end{array}$ \\
\hline Enero & 17,6 & 77 & 14,2 & 98 & 7,3 & 127,6 \\
\hline Febrero & 17,5 & 93 & 13,4 & 111 & 6,9 & 58,6 \\
\hline Marzo & 17,2 & 161 & 13,5 & 133 & 6,9 & 92,4 \\
\hline Abril & 16,9 & 113 & 13,4 & 91 & 6,9 & 141,2 \\
\hline Мayo & 16,3 & 62 & 12,7 & 42 & 7,1 & 146,4 \\
\hline Junio & 15,8 & 33 & 11,8 & 15 & 6,4 & 8,6 \\
\hline Julio & 15,4 & 20 & 11,9 & 8 & 6,2 & 5,4 \\
\hline Agosto & 15,7 & 37 & 12,3 & 14 & 6,2 & 3,4 \\
\hline Setiembre & 16,2 & 69 & 12,8 & 40 & 6,9 & 35,4 \\
\hline Octubre & 16,6 & 90 & 13,6 & 92 & 6,8 & 119,4 \\
\hline Noviembre & 16,8 & 69 & 13,3 & 68 & 8 & 146,6 \\
\hline Diciembre & 16,9 & 54 & 13,4 & 83 & 7,8 & 94,0 \\
\hline
\end{tabular}

Fuente: Gobierno Regional de Cajamarca y Estación Meteorológica Minera La Zanja para el año 2019.

\section{Aspectos principales de la propuesta de manejo mejorado}

La propuesta combina dos elementos que se refuerzan mutuamente. Por un lado, la renovación de pasturas, que promueven una mayor producción de pasturas de buena calidad. Al mismo tiempo una propuesta de modificar la utilización de este mayor forraje producido, acortando los turnos de pastoreo e introduciendo el manejo de realizar reservas de pasturas que se transfieren del periodo lluvioso al estiaje. La evaluación económica complementa, como debería ser, la viabilidad de la propuesta.

\section{Renovación de pasturas}

Se resembraron aquellas pasturas que estén invadidas por malezas entre 40 a $50 \%$, para ello se utilizó la yunta (utilización de animales de fuerza con el fin de labrar la tierra) o el tractor agrícola, que permite surcar paralelamente el suelo a una profundidad de 10 a $15 \mathrm{~cm}$.

En dichos surcos se colocaron semillas de raigrás “ecotipo cajamarquino" (15 kg/ha) y trébol blanco (3 $\mathrm{kg} / \mathrm{ha}$ ), además de urea (30 kg/ha) o abono orgánico y superfosfato triple $(80 \mathrm{~kg} / \mathrm{ha})$. Previamente se utilizó cal (2000 kg/ha) con el fin de reducir la acidez del suelo. La frecuencia de pastoreo se realizó cada 40 días y el excedente de la producción de pasturas que se genere durante la época de lluvia (noviembre-abril), fueron henificados y utilizados en la alimentación del ganado. Para ello se implementaron heniles, cubiertos con calamina, a fin de evitar la humedad.

Conservación de forrajes

Suponiendo que la cantidad de vacas en ordeño se mantienen una vez realizada la renovación de pasturas, existirá un superávit en el forraje producido durante el periodo de lluvia. Nuestra propuesta consiste en utilizar este excedente para elaborar reservas de forraje. Para ello, se ha elegido el método de henificación por deshidratación natural. Para que sea posible fue necesario contar con una infraestructura que evite que las lluvias, frecuentes durante este periodo, estén humedeciendo el material cortado, enlenteciendo el proceso y comprometiendo la calidad del producto final.

Se propuso construir un henil con materiales económi- 
cos, postes, tirantes, barras y hojas de calamina. El forraje cosechado fue almacenado bajo la cobertura del henil, requiriendo que el material fuera removido para homogenizar su proceso de secado. Una vez que se alcanzó el porcentaje de humedad apropiado (85 a 90\% de MS) el material fue comprimido en pacas de $40 \mathrm{~kg}$, para su posterior utilización en el periodo de estiaje.

\section{Evaluación económica}

Para la evaluación de la propuesta del manejo mejorado se usó la metodología de formulación y evaluación de proyectos (Pascale, 2009). Esta consiste en elaborar el flujo de fondos de la situación con proyecto ("manejo mejorado") y compararlo con el flujo de fondos ${ }^{1}$ de la situación sin proyecto ("manejo tradicional"). Ambos flujos de fondos representan los movimientos de fondos que ocurren en cada situación sobre la base de un ejercicio anual. Para la construcción de los flujos de fondos de ambas situaciones se utilizó un enfoque de "presupuesto parcial", el cual implica tener en cuenta únicamente los ingresos y egresos que cambian de una situación a otra, haciendo abstracción de todos los componentes financieros que se mantienen invariantes en ambas situaciones, por ejemplo los gastos en sanidad animal.

Así por el lado de los ingresos, se tuvo en cuenta los litros de leche que son producidos en una y otra situación. Al mismo tiempo, por el lado de los egresos, se tuvo en cuenta aquellos componentes que cambian entre una y otra situación, por ejemplo, el uso de la paja de arroz en el manejo tradicional y la cosecha y elaboración del heno de pastura en el otro.

La comparación entre ambas situaciones se realizó sobre la base de una hectárea de pastura. Se ha de suponer que en esta hectárea de pastura mantiene, en pastoreo, a tres vacas en lactancia. Durante un ejercicio anual este grupo de animales fue recorriendo las diferentes etapas del ciclo de la lactancia, sucediéndose los secados y partos de los animales.

Esta definición permitió delimitar los ingresos y egresos de ambas situaciones, así como el monto de las inversiones necesarias para realizar el mejoramiento de las pasturas y la construcción del henil.

\section{RESULTADOS Y DISCUSIÓN}

\section{Resultados de la comparación entre manejos}

Uso de insumos y resultados productivos para la situación de manejo tradicional y mejorado

La Tabla 2 contiene los coeficientes técnicos principales de los manejos tradicional y mejorado. La propuesta mejorada involucra renovación de una hectárea de pastura. Se supuso que del análisis de suelo realizado, surge la recomendación de corregir la acidez con encalado, aplicando $2000 \mathrm{~kg}$ cal/ha y fertilizar, utilizando $80 \mathrm{~kg}$ de superfosfato triple y 30 $\mathrm{kg}$ de urea.

Tabla 2. Consideraciones en el manejo tradicional y manejo mejorado

\begin{tabular}{ll}
\hline \multicolumn{1}{c}{ Manejo Tradicional } & \multicolumn{1}{c}{ Manejo Mejorado } \\
\hline 1 hectárea de pastura deteriorada & 1 hectárea de pastura deteriorada \\
3 vacas en ordeño (VO) & 3vacas en ordeño(VO) \\
Rendimiento promedio 6,5 litros/VO/día & Rendimiento promedio 9,75 litros/VO/día \\
Suplementación durante el periodo de estiaje: & Suplementación durante el periodo de estiaje: $4 \mathrm{~kg}$ de \\
$4 \mathrm{~kg}$ de paja de arroz/VO/día(92 días) & heno de pastura/VO/día(92 días) \\
\hline
\end{tabular}

\footnotetext{
${ }^{1}$ Un flujo de fondos son los movimientos de entrada y salida del efectivo, que generalmente ocurren cuando llevamos adelante un proceso de producción. Los movimientos de entrada o ingreso de efectivo constituyen las fuentes. De modo equivalente, los movimientos de salida o egreso de efectivo constituyen los usos del dinero. Los flujos de fondos se utilizan para evaluar la factibilidad de las prácticas de producción, así como para realizar evaluaciones cuantos estas son consideradas como formas alternativas de producir.

${ }^{2}$ La técnica de presupuesto parcial consiste en un método para analizar la conveniencia de realizar un cambio menor en el funcionamiento de un sistema de producción. Los cambios que pueden analizarse bajo este enfoque son diversos (de rubros similares, de insumos, o de la fuente de provisión de servicios), pero la característica común de estos cambios es que algunos componentes del sistema de producción, asociadas al cambio, se modifican y otras permanecen iguales en ambas situaciones. Estos últimos al mantenerse sin cambios pueden abstraerse del análisis. Por el contrario, los que, si cambian entre una situación y otra, serán el centro de nuestro análisis que evalúa los beneficios y costos del cambio.
} 
En la Tabla 3 se presenta el presupuesto de renovación de una hectárea de pasturas. De modo similar, la Tabla 4 expresa el presupuesto para la construcción de un henil con un tamaño mínimo para permitir la elabora- ción de la reserva forrajera (por henificación) necesaria para generar un suministro de $4 \mathrm{~kg}$ de heno de pradera durante 92 días del periodo de estiaje.

Tabla 3. Presupuesto considerado para la renovación de pasturas

\begin{tabular}{|c|c|c|}
\hline Renovación de pasturas perennes & Unitario(soles/unidad)(2) & 1 hectárea (soles) \\
\hline Análisis de suelo(2 muestras) & 35 & 70 \\
\hline $\begin{array}{c}\text { Fertilizante }(80 \mathrm{~kg} \text { de super fosfato } \\
\text { triple })(1)\end{array}$ & $105 / 50 \mathrm{~kg}$ & 168 \\
\hline Úrea $(30 \mathrm{~kg})(1)$ & $75 / 50 \mathrm{~kg}$ & 45 \\
\hline $\mathrm{Cal}(2000 \mathrm{~kg} / \mathrm{ha}$ y 3 jornales en & $13 / 50 \mathrm{~kg}$ & 610 \\
\hline aplicación)(1) & 30/jornal & \\
\hline Laboreo (2 pasadas de tiller, 3 horas/ha) & 80/hora & 240 \\
\hline Raigrás $10 \mathrm{~kg}$ & $15 / \mathrm{kg}$ semilla procesada & 150 \\
\hline Trébolblanco inoculado $2 \mathrm{~kg}$ & 30/kg semilla procesada y peleteada & 60 \\
\hline $\begin{array}{l}\text { Siembra y aplicación de fertilizante ( } 3 \\
\text { jornales por ha) }\end{array}$ & 30/jornal & 90 \\
\hline Total & & 1433 \\
\hline
\end{tabular}

(1): La fórmula de fertilización (cantidad y tipo) y encalado estará en función del análisis de suelo. La que se presenta asume un suelo promedio de la región de Cajamarca con muy poca historia de fertilización anterior.

(2): Los precios que se presentan fueron actualizados a mayo de 2020. (Tipo de cambio dólar: S/. 3,460).

Tabla 4. Presupuesto para la construcción de un henil de $50 \mathrm{~m}^{2}(1)$ para elaborar las reservas forrajeras en la escala de 1 hectárea de manejo mejorado.

\begin{tabular}{ccccc}
\hline Material & $\begin{array}{c}\text { Unidad de } \\
\text { medida }\end{array}$ & Cantidad & $\begin{array}{c}\text { Precio } \\
\text { (S/unidad)(3) }\end{array}$ & Total (S/.) \\
\hline Postes de eucalipto 3"x3"x10 ,5' & Unidad & 10 & 22 & 220 \\
Postes de eucalipto 2"x4"x16' & Unidad & 8 & 35 & 280 \\
Correas 1,5"x2"x10,5' & Unidad & 8 & 10 & 80 \\
Varas 1"x1 ,5"x10,5' & Unidad & 40 & 5 & 200 \\
Calamina 0,14 mm x 1 ,8 mx 0,8 m & Unidad & 56 & 9,9 & 554,4 \\
Clavos 2" & kg & 2 & 4,9 & 9,8 \\
Clavos de calamina 2,5"x50 und & Bolsa & 9 & 30 & 30,1 \\
Trabajo(2) & Jornal & 10 & & $\mathbf{1 7 0 6 , 3}$ \\
\hline Total & & &
\end{tabular}

(1): El dimensionamiento del henil esta en relación a la necesidad de producir el suficiente heno para suplementar a las 3 VO a razón de 4 kg por día durante los 92 días del estiaje. Suponiendo un heno de $85 \%$ de MS y pérdidas durante el proceso de $25 \%$, es necesario reservar una superficie de $1000 \mathrm{~m}^{2}$ de pastura durante 120 días para realizar 3 cortes que produzcan al menos los $1104 \mathrm{~kg}$ de heno necesario. Se supone una producción de 2 $\mathrm{kg}$ de pastura por $\mathrm{m}^{2}$ en un periodo de 40 días durante la época de lluvia con la pastura renovada. Los cortes se irán escalonando para optimizar la superficie de secando disponible.

(2): Seguramente sea necesario dos tipos de trabajo, un constructor y trabajo menos especializado (peones) que seguramente tenga un valor diferente.

(3): Los precios que se presentan fueron actualizados a mayo de 2020 (Tipo de cambio dólar: S/.3,460).

Flujo de fondos para la situación de manejo tradicionalymejorado

Las siguientes tablas presentan los flujos de fondos, del manejo tradicional o "situación sin proyecto" (Tabla 5); del manejo mejorado o "situación con proyecto" (Tabla 6) y otros dos combinando los dos primeros. La Tabla 7 presenta el flujo incremental sin incluir el valor de rescate de las inversiones no amortizadas y la Tabla 8 presenta el flujo incremental con ese valor de recuperación del capital invertido.
Tabla 5. Flujo de fondos del manejo tradicional.

\begin{tabular}{cc}
\hline Manejo tradicional(1) & Año i \\
\hline Ingreso (soles/año)(2) & 7118 \\
Costo(soles/año)(3) & 850 \\
Saldo(soles/año) & 6267 \\
\hline
\end{tabular}

(1): Elaborado con los coeficientes técnicos definidos en la Tabla 2.

(2): Precio de la leche, 1 sol por litro.

(3): Precio de la paja de arroz, 0.77 soles por $\mathrm{kg}$ (pacas de $20 \mathrm{~kg}$ ). 
Tabla 6. Flujo de fondos del manejo mejorado

\begin{tabular}{lcc}
\hline Manejo mejorado(1) & Año 0 & Año 1 \\
\hline Inversiones(2) & 3139 & 10676 \\
Ingreso(soles/año)(2) & \\
Costos & & 405 \\
Cosecha de forraje y elaboración del heno(3) & 85 \\
Mantenimiento del henil(4) & 143 \\
Mantenimiento de la pradera(5) & -3139 & 10043 \\
Saldo & & \\
\hline
\end{tabular}

(1): Elaborado con los coeficientes técnicos definidos en la Tabla 3.

(2): elaborado a partir de las Tablas 4 y 5 . Las inversiones por definición se realizan en el año " 0 ".

(3): Para la cosecha y acarreo del forraje a conservar se utilizan 3 jornadas por corte, sumando para los 3 cortes un total de 9 jornadas; el secado y empacado del forraje insume 1.5 jornada por cada corte, en total 4.5 jornadas. En suma 13.5 jornales, a 30 soles el jornal, 405 soles

(4): se supone un $5 \%$ del valor a nuevo como costo de reparación y mantenimiento del henil.

(5): aunque esta partida no es estrictamente necesaria, proponemos formar un fondo de reinversión para el mejoramiento de las pasturas, de modo que la propuesta de manejo mejorado adquiera una naturaleza sustentable.

Tabla 7. Flujo de fondos incremental sin recuperación del capital

\begin{tabular}{ccc}
\hline Items & Año 0 & Año 1 \\
\hline Saldo MT(soles/año) & & 6267 \\
Saldo MM(soles/año) & -3139 & 10043 \\
Saldo incremental(soles/año) & -3139 & 3776 \\
\hline
\end{tabular}

En el año "0" los saldos de ambos modelos de manejo son iguales. También es el periodo cuando se realizan las inversiones. Como fue mencionado, la renovación de las pasturas se realiza en octubre-noviembre. Se estima que deberá esperarse un periodo de unos 70 días para volver a iniciar los pastoreos en esa hectárea

Tabla 8. Flujo de fondos incremental con recuperación del capital

\begin{tabular}{ccc}
\hline Ítems & Año 0 & Año 1 \\
\hline Saldo MT(soles/año) & & 6267 \\
Saldo MM con recuperación del capital(soles/año)(1) & -3139 & 12868 \\
Saldo incremental (soles/año) & -3139 & 6601 \\
\hline
\end{tabular}

(1): al finalizar el primer año en la situación con manejo mejorado, además de la mayor producción de leche se dispondrá de una hectárea de pastura mejorada y de un henil, ambos con una vida útil de 9 años remanente, activos que podemos valorizar al $90 \%$ de su costo de producción.

\section{Evaluación financiera y económica}

Desde el punto de vista financiero, la propuesta de manejo mejorada genera un saldo incremental que permite repagar las inversiones necesarias para la implementación del mismo durante el primer año de aplicación del proyecto. La tasa interna de retorno (TIR) sin recuperación del capital es de $20 \%$, mientras que la TIR con recuperación del capital es de $110 \%$. Adicionalmente en ese primer año se genera un fondo de reserva para mantener los mejoramientos de pasturas equivalentes al 10\% del valor de dicha inversión, es decir, de 143 soles.

Evaluación de las ventajas productivas y económicas del manejo mejorado
Se puede afirmar que el aumento de frecuencia del pastoreo (de 75 a 40 días), asociado a la renovación de pasturas, fertilización, y a la conservación de forraje para suplementar a las vacas en lactancia, durante el estiaje, es un cambio de manejo que está bien respaldado por el comportamiento productivo y nutricional de las pasturas (Vallejos 2009; Yu Ying y Hou Fu, 2005; Silveira y Kohmann, 2020; Marino y Agnusdei, 2004; Álvarez, 2006; García, 2006; Vallejos, 2017; Vallejos et al., 2019; Carrasco, 2019; Vallejos, 2011) y por los datos financieros y económicos del estudio.

La implementación de este manejo mejorado requiere una inversión por ha, de 3139 soles y hay un aumento en los requerimientos en trabajo de 19,5 jornadas en el 
año. Este aumento en los requerimientos de trabajo surge de las labores de encalado (3 jornadas), de la fertilización y siembra (3 jornadas), del corte y acarreo del forraje para conservar (9 jornadas) y del acondicionamiento y empacado del heno de pradera (4,5 jornadas). A estas también pueden sumarse parte de las jornadas necesarias para la construcción del henil, en caso de disponer de las capacidades necesarias, pudiéndose alcanzar un total de incremento para el $1^{\circ}$ año de unos 25 jornales. La propuesta de manejo mejorado entonces, agrega una demanda de trabajo equivalente a un mes de empleo completo por hectárea de pastura mejorada, equivalente a 750 soles de ingreso que se pueden sumar al ingreso familiar en caso de que el trabajo sea realizado por el productor y su familia. Esta cantidad de trabajo ha sido computada como costo de la propuesta de manejo mejorado incluida en la inversión, utilizando un valor de mercado para remunerar este tipo de trabajo y es valor que puede mejorar el ingreso familiar. En solo el primer año, el incremento en los ingresos es capaz de financiar el costo de las inversiones en la renovación de pasturas y la construcción de un henil para el secado y conservación de las reservas de forraje.

La diferencia de ingresos entre los manejos, medida a través del saldo incremental, permite financiar las inversiones con un costo de hasta $20 \%$ de tasa efectiva anual (TEA). Cuando tenemos en cuenta el valor por la recuperación del capital invertido en la propuesta, la TIR del proyecto aumentó hasta un valor de $110 \%$.

Sobre los aspectos de la propuesta que pueden promover o enlentecer su implementación a nivel de productor

Inversión para la renovación de pasturas permanentes $y$ elaboración de reservas forrajeras y su financiamiento La implementación de esta propuesta demandó la realización de dos inversiones importantes, la renovación de pasturas y el henil para la elaboración de la reserva forrajera. Aún, bajo el supuesto de que el productor y su familia aportaron la mayor parte del trabajo necesario (distribución de la cal, distribución del fertilizante y semilla y colaboración en la construcción del henil), se contó con un monto de 2800 soles por hectárea incorporada al nuevo manejo. Para los productores pequeños y medianos es un monto importante, equivalente al valor de casi 4 meses de remuneración de un trabajador rural. Un resultado similar obtenemos al comparar este monto de inversión con el ingreso familiar promedio reportado por Perinango (2019), para una muestra de 272 ganaderos del Centro Poblado Huacataz, en el distrito de Baños del Inca. En este caso el monto de la inversión representó el 32,8\% del ingreso familiar promedio.

Difícilmente los pequeños y medianos productores disponen de ahorros familiares para poder canalizar la propuesta, con la finalidad de mejorar sus sistemas de producción. En tal sentido, fue necesario contar con el sistema bancario que facilitó créditos para promover este desarrollo. Fue deseable que los plazos y otras condiciones se adecuen con la capacidad de repago que genera la inversión de pasturas asociada a la elaboración de reservas. Si bien el retorno de la inversión es rápido, se entiende conveniente trabajar con un periodo de repago de al menos dos años.

\section{Disponibilidad de servicios de maquinaria}

Debido a la micro-escala predominante en los fundos, la inmensa mayoría de los ganaderos de la región de Cajamarca carecen de equipos o animales para trabajar la tierra. Debió promoverse la disposición de contratistas que ofrecieron servicios de laboreo a precios competitivos.

Prácticas de conservación de forraje, elaboración de heno en el periodo lluvioso.

No existe entre los ganaderos de Cajamarca una cultura de elaboración de reservas forrajeras, por lo que se debió crear. Para ello es importante el desarrollo de experiencias demostrativas. Los fundos que son manejados por instituciones de enseñanza e investigación juegan un papel importante, realizando actividades demostrativas, invitando a productores y sus familias a observar y participar en las mismas.

Aumento en la demanda de trabajo

Como fuera mencionado, la implementación de una hectárea bajo un nuevo manejo, genera una demanda 
adicional de al menos unas 20-25 jornadas de trabajo rural durante el $1^{\circ}$ año de implementación del nuevo manejo. A partir del $2^{\circ}$ año, el incremento se estabiliza en unas 10 jornadas; muchos productores podrán satisfacer este requerimiento a partir del recurso laboral familiar subutilizado. En otras circunstancias, se deberá contratar trabajo extra-predial. En el primer caso, la propuesta de manejo mejorado, además de contribuir al ingreso familiar con una mayor producción de leche, aportó un salario mensual extra a la economía predial durante el primer ejercicio, para luego estabilizarse en medio salario mensual. En el segundo caso la propuesta contribuirá a aumentar el empleo y aumentar los ingresos de la comunidad local.

\section{CONCLUSIONES}

La resiembra y fertilización en pasturas degradadas permitieron recuperar el rendimiento óptimo de las mismas, evitando el sobrepastoreo, y manteniendo o incrementando la carga animal actual.

Acortando los periodos de frecuencias de pastoreo tradicionales, se mejoró el valor nutricional de las pasturas y consecuentemente el rendimiento de leche. Diferir los excedentes forrajeros desde el periodo de lluvia al periodo de estiaje mediante la realización de reservas forrajeras permite balancear la producción de forrajes con los requerimientos alimenticios de los animales, permitiendo alcanzar lactancias más largas y productivas.

Los ingresos económicos de las familias rurales se vieron incrementados mejorando su nivel de vida. También se generó una demanda de trabajo y servicios que redunda en mayores ingresos para la comunidad rural.

\section{REFERENCIAS BIBLIOGRÁFICAS}

Álvarez, M. 2006. El impacto de la siembra directa en los campos de cria 2016. h t t p : / / w w w. p r o d u c c i o n animal.com.ar/informacion_tecnica/cria/93 impacto_siembra_directa.pdf (Consultada el 30 de junio de 2020)
Carrasco, W. 2019. Determinación del estado actual de la composición florística del piso forrajero en la campiña de Cajamarca. Tesis de Maestría. Universidad Nacional de Cajamarca. Cajamarca (Perú).

García, F. 2006. El rol del fósforo en la producción de pasturas de la región pampeana 2016. h t t p : / / w w w. p r o d u c c i o n animal.com.ar/produccion_y_manejo_pastur as / pasturas_fertilizacion/21 fosforo_en_pasturas.pdf(Consultada el $30 \mathrm{de}$ junio de 2020).

Marino, M. y M. Agnusdei. 2004. Conceptos básicos para el manejo de la nutrición nitrogenada y fosfatada de las pasturas. $2^{a}$ Jornada de Actualización. http://www.produccion nimal.com.ar/produccion_y_manejo_pastura s/pasturas_fertilizacion/0 3 mutricion_nitrogenada_fosfatada.pdf (Consultada el 30 de junio de 2020).

Pascale, R. 2009. “Decisiones Financieras”. Buenos Aires (Argentina): Pearson.

Perinango, J. 2019. Relación entre sistema ganadero y sostenibilidad agropecuaria, social y económica en el Centro Poblado Huacataz, distrito de Baños del Inca”. Tesis de Maestría. Universidad Nacional de Cajamarca. Cajamarca (Perú).

Silveira, M. L., y M. M. Kohmann. 2020. “Chapter 3 Maintaining soil fertility and health for sustainable pastures." En Management Strategies for Sustainable Cattle Production in Southern Pastures. Rouquette, M. y G. E. Aiken (eds). Londres (Reino Unido): Academic Press. DOI:10.1016/b978-0-12814474-9.00003-7

Vallejos, L. A. 2009. Efecto de la fertilización fosforada y frecuencia de pastoreo sobre el valor nutritivo de la dieta y comportamiento ingestivo de las vacas Holstein en pasturas de ryegrass-trébol en Cajamarca. Tesis de Doctorado. Universidad Nacional Agraria La Molina. Lima (Perú). 
Vallejos, L. A. 2017. Investigación en la introducción de especies forrajeras neozelandesas capaces de proporcionar elevados rendimientos de alta calidad para el mejoramiento del volumen de producción de leche y venta de quesos de la asociación de Pucará El Trébol en Santa Cruz-Cajamarca-Perú. Informe Final. Lima (Perú)

Vallejos, L. A., R. R. Florián, J. Perinango, P. Barboza. 2011. Programa de alimentación basado en pasturas, orientado a la conservación del genotipo rye grass ecotipo cajamarquino $e$ incremento de la producción del bovino lechero, en dos pisos altitudinales de la región Cajamarca. Informe. Cajamarca (Perú)

Vallejos, L. A., I. B. Rojas, J. Perinango, J., Alcántara, J. 2019. "Vacas pastoreadas a estaca y su efecto sobre el consumo y condición de la pastura." UCV - Scientia 11 (1): 28-31. DOI: 10.18050/ucv-scientia.v11i1.2400

Yu Ying, W. y J. Hou Fu. 2005. "Cutting effects on growth characteristics, yield composition, and population relationships of perennial ryegrass and white clover in mixed pasture." New Zealand Journal of Agricultural Research 48 (3): 349-358 